\title{
Avaliação de protocolo de fisioterapia aplicado a pacientes mastectomizadas a Madden
}

\author{
Evaluation of a physiotherapeutic protocol applied to mastectomized patients
}

\author{
Carla Maria de Abreu Pereira물 Elidia Orie Rodrigues Yamada Vieira², Paulo Sérgio Martins Alcântara ${ }^{3}$
}

\begin{abstract}
Resumo
O bjetivo: Avaliação dos resultados da aplicação de um protocolo de fisioterapia aplicado em pacientes mastectomizadas (tipo M adden). M étodos: Foram avaliadas 119 pacientes, com idade entre 29 e 79 anos, submetidas a M astectomia M odificada R adical T ipo M adden, no Instituto de M astologia e G inecologia do H ospital Beneficência Portuguesa - São Paulo, durante o período de maio/2001 a dezembro/2002. D estas 119 pacientes, apenas 44 foram selecionadas de acordo com critérios de inclusão e exclusão. Formaram-se 2 grupos, sendo um grupo com 33 pacientes que foram submetidas a um protocolo fisioterapêutico até a alta e um outro, com 11 pacientes que desistiram do tratamento. A amplitude de movimento (ADM ) e uma possível evolução para linfedema foram avaliados em ambos os grupos. Resultados: Todas as pacientes submetidas ao protocolo fisioterapêutico, ao final do tratamento estavam sem limitação de ADM e/ou com pequena limitação, com uma faixa de variação de $150^{\circ}$ a $180^{\circ}$, e apenas 3 evoluíram para linfedema. Em relação ao grupo desistente do tratamento, na reavaliação a limitação de $A D M$ variou de $40^{\circ}$ a $170^{\circ}$, sendo que 4 pacientes evoluíram para linfedema. Conclusão: 0 protocolo apresentado neste estudo mostrou-se eficaz para as pacientes pós-mastectomizadas. Conclui-se também que a intervenção precoce da fisioterapia, aplicada ainda no ambiente hospitalar, não só ajuda a prevenir as complicações pós-cirúrgicas, como também reabilita as pacientes mais cedo para as atividades da vida diária (AVDs), e ainda, permite a elas que possam se valer da colaboração e do incentivo da equipe multidisciplinar para o tratamento. Palavras-chave: N eoplasias mamárias; Fisioterapia; M astectomia radical modificada de M adden; Linfedema.
\end{abstract}

${ }^{1}$ Fisioterapeuta, membro da Equipe de Fisioterapia do Instituto de Ginecologia e M astologia do H ospital Beneficência Portuguesa

${ }^{2}$ Fisioterapeuta, chefe de equipe de fisioterapia do Instituto de Ginecologia e M astologia do H ospital Beneficência Portuguesa

${ }^{3}$ M S Cirurgião oncológico, Assistente da Divisão de Clínica Cirúrgica do H ospital Universitário da Universidade de São Paulo.

Endereço para correspondência: Ft. Carla M aria de Abreu Pereira. E-M ail: fisiocarla@itelefonica.com.br 


\begin{abstract}
O bjective The objective of this study was to assess a physiotherapeutic protocol applied to mastectomized patients ( $M$ adden's technique). M ethods: 119 patients aged 29-79 years were assessed and submitted to M odified Radical $M$ astectomy by $M$ adden'stechnique, at the Instituto de $M$ astologia $\mathrm{G}$ inecologia do $\mathrm{H}$ ospital Beneficência Portuguesa, São Paulo, from M ay 2001 to D ecember 2002. O ut of these 119 patients, only 44 were selected according to inclusion and exclusion criteria. Two groups were composed, one with 33 patients submitted to a physiotherapeutic protocol up to discharge, and another group with 11 patients who discontinued treatment. ROM and likely progression to lymphedema were assessed in both groups. Results. By the end of treatment, all patients submitted to the physiotherapeutic protocol presented no limitation and/or small limitation in ROM, varying from $150^{\circ}$ to $180^{\circ}$, and only three of them devel oped lymphedema. When the group who discontinued treatment was reassessed, ROM limitation varied from $40^{\circ}$ to $170^{\circ}$, and four patients developed Iymphedema. Conclusion: This study showed the efficiency of the proposed protocol for post mastectomized patients. Early physiotherapeutic intervention applied at hospital, not only helps preventing post-surgical complications, but it also rehabilitates patients earlier for their activities of daily living (ADLs). M oreover, it benefits them by receiving collaboration and encouragement for treatment provided by a multidisciplinary team.

Key words. Breast neoplasms; Physi otherapy; M odified radical mastectomy by M adden's technique; Lymphedema.
\end{abstract}

\section{INTRO DUÇÃO}

0 câncer de mama vem ocupando lugar de destaque, por apresentar incidência crescente e elevado índice de mortalidade. $\mathrm{N}$ ão é apenas 0 mais comum entre as mulheres, mas também é o que mais mata. $\mathrm{N}$ os Estados Unidos são diagnosticados 180 mil novos casos por ano e cerca de 44 mil mulheres morrem de câncer de mama todos os anos. No Brasil, o câncer de mama é o que mais causa mortes entre as mulheres. Em 1999, foram registradas 8.104 mortes decorrentes deste tipo de câncer. D os 337.535 novos casos de câncer com previsão de serem diagnosticados em 2002, o câncer de mama foi o principal a atingir a população feminina, sendo responsável por 36.090 novos casos. ${ }^{1-5}$

0 carcinoma ductal éo tumor mamário mais comum e torna-se invasivo em cerca de $70 \%$ dos casos, dando origem a metástases ganglionares em $1 \%$ a $13 \%$ dos casos. $^{6-8} 0$ tratamento é planejado de acordo com 0 estadiamento da doença e a cirurgia é parte importante para o tratamento câncer localizado.9-12

A mastectomia radical modificada de $M$ adden consiste na remoção da glândula mamária, aponeurose anterior e posterior do músculo grande peitoral, esvaziamento axilar, com preservação dos músculos grande e pequeno peitoral; e indicada para tumores de $2 \mathrm{~cm}$ a $3 \mathrm{~cm}$ ou inferiores a $2 \mathrm{~cm}$, desde que o carcinoma seja invasivo, e pode ter como complicações a limitação de amplitude de movimento (ADM), limitação de função, linfedema e dor no membro homolateral. . $^{13-17}$

Para estes casos, a fisioterapia vem demonstrando ser fundamental quando iniciada precocemente em mulheres mastectomizadas. 0 tratamento fisioterapêutico tem como objetivos, controlar a dor no pós- operatório, prevenir ou tratar linfedema e alterações posturais, promover o relaxamento muscular, manter a amplitude de movimento do membro superior envolvido (o mais próximo de $180^{\circ}$ deflexão e abdução da articulação glenoumeral), melhorar 0 aspecto e maleabilidade da cicatriz, prevenindo ou tratando as aderências. 0 recurso terapêutico utilizado é a cinesioterapia (que consiste na reabilitação através de exercícios) e orientações para as atividades de vida diária. ${ }^{18,19}$

\section{MÉTO DOS}

Foram avaliadas 119 pacientes, com idade entre 29 e 79 anos, submetidas a M astectomia M odificada Radical Tipo Madden, no Instituto Ginecologia e $M$ astologia (IG M ) do H ospital Beneficência Portuguesa - São Paulo, durante o período de maio/2001 a dezembro/2002. D estas 119 pacientes, apenas 44 foram selecionadas de acordo com os seguintes critérios de inclusão e exclusão:

- Critérios de inclusão: ser portadora de câncer de mama ductal; ter sido submetida à mastectomia radical modificada tipo $\mathrm{M}$ adden na mama esquerda ou direita; apresentar limitação de amplitude articular (flexão e abdução de ombro) pós-mastectomia do membro superior homolateral; ter realizado 28 sessões de radioterapia; ter recebido orientação médica para efetuar tratamento fisioterapêutico pós-operatório e ter sido 
submetida a avaliação inicial e final ao tratamento.

- Critérios de exclusão: ter sido submetida a qualquer outro tipo de cirurgia da mama, que não a do tipo $M$ adden; ser portadora de outros tipos de carcinomas, que não o ductal; ter sido submetida a mastectomia bilateral; ser portadora de qualquer outra patologia que poderia comprometer a ADM do membro superior homolateral à cirurgia (como por exemplo: síndrome do impacto, bursite etc).

Todas as pacientes selecionadas foram avaliadas por goniometria (método que avalia os ângulos articulares através do uso de um goniômetro) e perimetria (método que avalia a circunferência dos membros, para verificar possíveis alterações), antes e ao término do tratamento fisioterapêutico.

Todas as pacientes compareceram à fisioterapia no mesmo período pós-cirúrgico (uma semana após a cirurgia), e foram submetidas na $1^{\text {a }}$ sessão de fisioterapia a uma avaliação que constava de anamnese, goniometria e perimetria (na posição ortostática); além disso, receberam orientação para a prática de exercícios, através da entrega do manual fisioterapêutico para pósoperatório em pacientes submetidas à mastectomia. ${ }^{20-21}$

0 protocolo de tratamento fisioterapêutico foi aplicado duas vezes por semana, em sessões de 45 minutos de duração, tendo sido desenvolvido e aplicado da seguinte forma, a partir da $2^{\text {a }}$ sessão: interrogatório; cinesioterapia (alongamento passivo demembro superior - M S, mobilização escapular, exercícios passi vos de flexão e de abdução de ombro, exercícios ativos assistidos de flexão e abdução de ombro); pompage: manobra que atua na fáscia muscular ${ }^{22}$ (global; pompage dos músculos: trapézio, peitoral maior e menor; pompage de ombro); posicionamento do membro superior homolateral à cirurgia. A partir da $3^{\text {a }}$ e $4^{\text {a }}$ sessões: interrogatório; cinesioterapia (alongamento M S, mobilização escapular, exercícios passivos de abdução de ombro, exercícios ativos assistidos, de flexão e de abdução de ombro; pompage (global, pompage dos músculos: trapézio, peitoral maior e menor, pompage de ombro); mobilização da cicatriz, orientação e demonstração da auto-massagem. A partir da $5^{\text {a }}$ sessão: interrogatório; cinesioterapia (mobilização escapular, exercícios ativo livres, de flexão e de abdução de ombro, mobilização da cicatriz); pompage (global; pompage dos músculos: trapézio, peitoral maior e menor; pompage de ombro); orientação e demonstração da auto-massagem.

A goniometria foi definida arbitrariamente em 3 graus de severidade de comprometimento da ADM (flexão e abdução) do membro superior homolateral à cirurgia, baseados na função das atividades da vida diária (AVD s). Assim, têm-se: de $0^{\circ}$ a $60^{\circ}$ - grau severo, $61^{\circ}$ a $120^{\circ}$ - grau moderado, $121^{\circ}$ a $180^{\circ}$ - grau leve ou comprometimento mínimo.

A medida de referência do método da perimetria para avaliação de linfedema foi de $7 \mathrm{~cm}$, partindo-se do ponto fixo da prega do cotovelo, sendo $7 \mathrm{~cm}, 14 \mathrm{~cm}$ e $21 \mathrm{~cm}$ acima e abaixo desta prega, mais a medida da circunferência da mão.

\section{CASUÍSTICA}

D entre as 44 pacientes selecionadas de acordo com os critérios de inclusão e exclusão, 33 realizaram 0 tratamento fisioterapêutico até receberem alta (Grupo A) e 11 desistiram do tratamento fisioterapêutico, após a $1^{\text {a }}$ sessão, por motivos pessoais, formando assim um grupo de desistentes (G rupo B).

0 grupo que desistiu do tratamento (Grupo B) foi convocado para uma nova avaliação.

Os dois grupos de pacientes (A e B), por sua vez, foram divididos em três subgrupos cada um para o estudo do membro do lado operado de acordo com a severidade do comprometimento pós-tratamento cirúrgico avaliado pela goniometria.

0 protocolo de tratamento fisioterapêutico foi seguido, respeitando o limite de cada paciente e todas pacientes após a $4^{a}$ sessão, foram orientadas a realizarem duas vezes por dia, em casa, os exercícios ativo livres do manual fisioterapêutico para pós-operatório em pacientes submetidas à mastectomia.

A avaliação dos dados foi realizada através do software W inST AT The Statistics Program for Windows. ${ }^{23}$

\section{RESULTADO S}

Em relação à limitação da $A D M$ do movimento de flexão da articulação gleno-umeral, na $1^{\text {a }}$ sessão do grupo A a variação foi de $20^{\circ}$ a $140^{\circ}$, com média de $85,45^{\circ}$ e do grupo $B$ a variação foi de $50^{\circ}$ a $140^{\circ}$, com média de $82^{\circ}$. Por ocasião da alta, a variação de limitação da AD M do grupo $A$ foi de $150^{\circ}$ a $180^{\circ}$, com média de $173,75^{\circ}$, e do grupo $B$ foi de $140^{\circ}$ a $170^{\circ}$, com média de $127,72^{\circ}$.

Q uanto à limitação da ADM do movimento de abdução da articulação gleno-umeral, na $1^{\text {a }}$ sessão do grupo $A$ a variação foi de $10^{\circ}$ a $140^{\circ}$, com média de $75,75^{\circ}$ e do grupo B a variação foi de $30^{\circ}$ a $120^{\circ}$, com média de $66,81^{\circ}$. Por ocasião da alta, a variação de limitação da ADM do grupo A foi de $150^{\circ}$ a $180^{\circ}$, com média de $174,84^{\circ}$, e do grupo $B$ foi de $50^{\circ}$ a $170^{\circ}$, com média de $120,90^{\circ}$.

D as 33 pacientes do grupo A, 3 evoluíram para linfedema, representando $9,09 \%$ da população e, das 11 pacientes do grupo B, 4 evoluíram para linfedema, 
representando 36,36\% das pacientes.

$N$ este estudo também foi possível observar que as pacientes do grupo A que, na $1^{a}$ avaliação, não atingiam 1800 de flexão e abdução da articulação gleno-umeral do membro superior contra-lateral à cirurgia, obtiveram um ganho total, na maioria dos casos, do grau de flexão e abdução.

O s resultados de ambos os grupos foram tabulados (Tabelas 1 a 4), resultando nas respectivas análises descritivas, enas figuras apresentados em seguida (Figuras 1 a 4), para cada grupo estudado. $N$ as Figuras 1 e 2, a área preenchida corresponde à maior concentração de pacientes na faixa de valores e a linha cheia apresentada dentro desta área corresponde à mediana.

Tabela 1 - Grupo A: Análise descritiva da avaliação inicial ( $1^{\text {a }}$ sessão) e final (alta) da flexão da articulação gleno-umeral.

\begin{tabular}{l|c|c}
\hline Número de pacientes & $\begin{array}{c}\text { Grupo A } \\
1^{\text {a }} \text { sessão }\end{array}$ & $\begin{array}{c}\text { Grupo A } \\
\text { Alta }\end{array}$ \\
\hline Média & 33 & 33 \\
\hline Mediana & 85,45 & 173,75 \\
\hline Valor mínimo & 80 & 177,50 \\
\hline Valor máximo & 20 & 150 \\
\hline Amplitude total (faixa) & 140 & 180 \\
\hline
\end{tabular}

Tabela 2 - G rupo B: Análise descritiva da avaliação inicial (1 $1^{\text {a }}$ sessão) e reavaliação da flexão da articulação gleno-umeral.

\begin{tabular}{l|c|c}
\hline & $\begin{array}{c}\text { Grupo B } \\
1^{\text {a }} \text { sessão }\end{array}$ & $\begin{array}{c}\text { Grupo B } \\
\text { Reavaliação }\end{array}$ \\
\hline Número de pacientes & 11 & 11 \\
\hline Média & 82 & 127,72 \\
\hline Mediana & 80 & 140 \\
\hline Valor mínimo & 50 & 40 \\
\hline Valor máximo & 140 & 170 \\
\hline Amplitude total (faixa) & 90 & 130 \\
\hline
\end{tabular}

Tabela 3 - G rupo A: Análise descritiva da avaliação inicial (1 ${ }^{\text {a }}$ sessão) e final (alta) da abdução da articulação gleno-umeral.

\begin{tabular}{l|c|c}
\hline Número de pacientes & $\begin{array}{c}\text { Grupo A } \\
1^{\text {a }} \text { sessão }\end{array}$ & $\begin{array}{c}\text { Grupo A } \\
\text { Alta }\end{array}$ \\
\hline Média & 33 & 33 \\
\hline Mediana & 75,75 & 174,84 \\
\hline Valor mínimo & 80 & 180 \\
\hline Valor máximo & 10 & 150 \\
\hline Amplitude total (faixa) & 140 & 180 \\
\hline
\end{tabular}

Tabela 4 - G rupo B: Análise descritiva da avaliação inicial (1ª sessão) e reavaliação da abdução da articulação gleno-umeral.

\begin{tabular}{l|c|c}
\hline & $\begin{array}{c}\text { Grupo B } \\
1^{\text {a }} \text { sessão }\end{array}$ & $\begin{array}{c}\text { Grupo B } \\
\text { Reavaliação }\end{array}$ \\
\hline Número de pacientes & 11 & 11 \\
\hline Média & 66,81 & 120,90 \\
\hline Mediana & 70 & 140 \\
\hline Valor mínimo & 30 & 50 \\
\hline Valor máximo & 120 & 170 \\
\hline Amplitude total (faixa) & 90 & 120 \\
\hline
\end{tabular}

A Figura 1 mostra a análise descritiva da flexão da articulação gleno-umeral, entre os grupos A e B. N o início do tratamento ( $1^{\text {a }}$ sessão) a limitação de ADM do grupo A oscilava de $20^{\circ}$ a $140^{\circ} \mathrm{com}$ uma média igual a $85,45^{\circ}$, e a do grupo B oscilava de $50^{\circ}$ a $140^{\circ}$, com média igual a $82^{\circ}$. Já na ocasião da alta do grupo $A, a$ oscilação era de $150^{\circ}$ a $180^{\circ}$, com uma média de $173,75^{\circ}$. $\mathrm{N}$ a reavaliação do grupo $\mathrm{B}$, a oscilação situava-se na faixa de $50^{\circ}$ a $170^{\circ}$, com uma média de $127,72^{\circ}$.

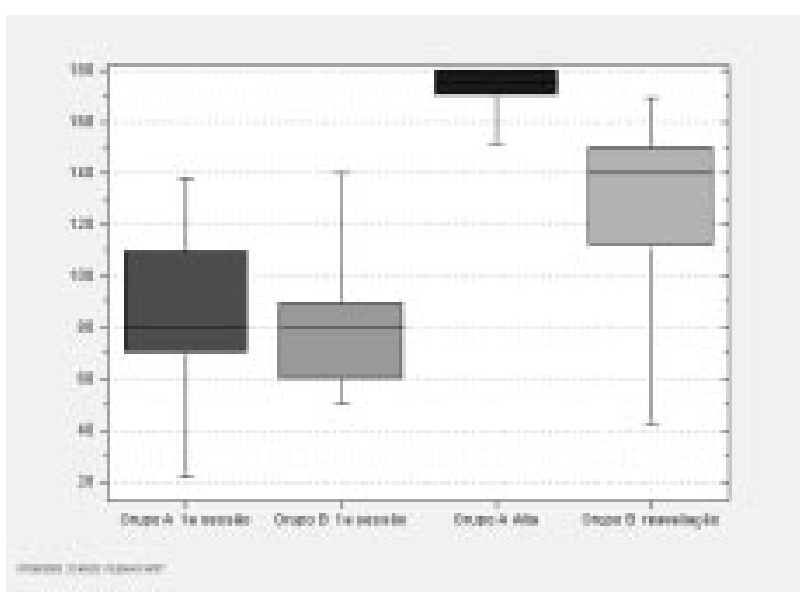

Figura 1 - Análise descritiva da flexão da articulação glenoumeral, entre os grupos A e B.

A Figura 2 mostra a análise descritiva da abdução da articulação gleno-umeral, entre os dois grupos $A$ e $B$. $\mathrm{N}$ o início do tratamento ( ${ }^{\mathrm{a}}$ sessão) a limitação de AD M do grupo A oscilava de 100 a 1400 , com uma média igual a $75,75^{\circ}$, e a do grupo B oscilava de $30^{\circ}$ a $120^{\circ}$, com média igual a $66,81^{\circ}$. Já na ocasião da alta do grupo A, a oscilação era de $150^{\circ}$ a $180^{\circ}$, com uma média de $174,84^{\circ}$. N a reavaliação do grupo $B$, a oscilação situavase na faixa do $50^{\circ}$ a $170^{\circ}$, com uma média de $120,90^{\circ}$.

A Figura 3 mostra que apenas 9,09\% (3 pacientes) apresentaram evolução para linfedema durante 0 tratamento, enquanto na Figura 4 observa-se que no grupo B 36\% (4 pacientes) apresentaram evolução para linfedema. 


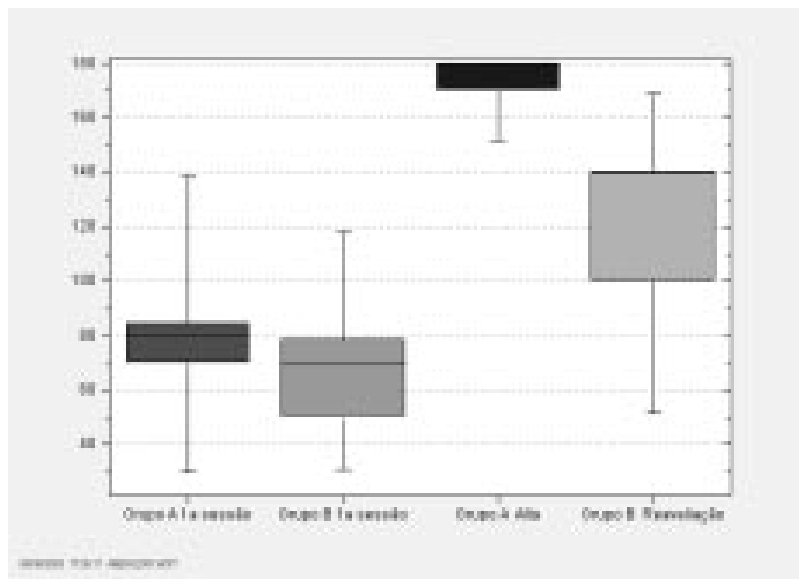

Figura 2 - Análise descritiva da abdução da articulação glenoumeral, entre os grupos A e B.

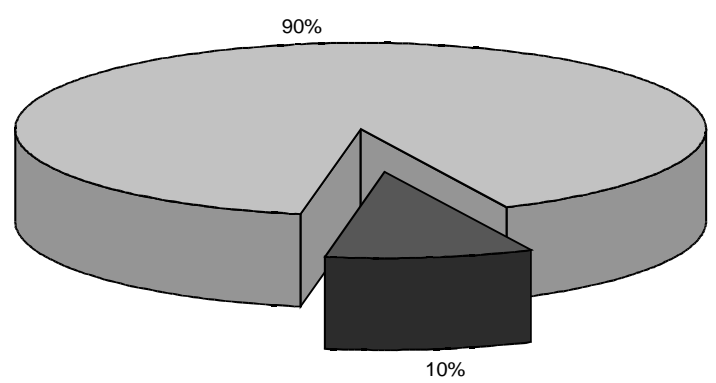

口SEM EVO LUÇÃO

ロCOM EVOLUÇÃO

Figura 3 - Percentual de pacientes no grupo A com evolução para linfedema durante 0 tratamento.

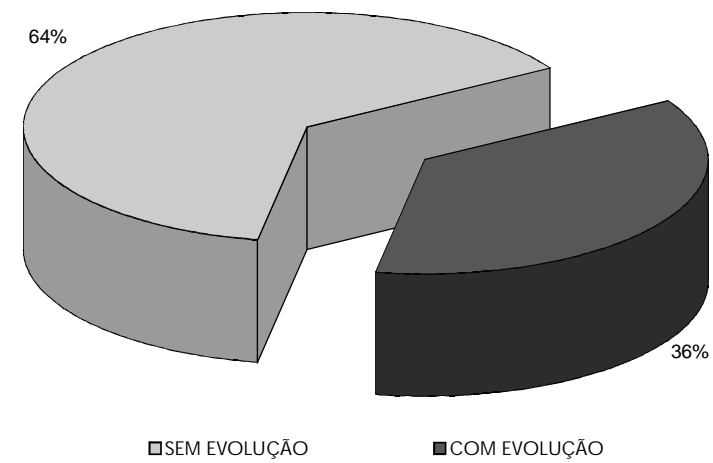

Figura 4 - Percentual de pacientes no grupo B com evolução para linfedema durante 0 tratamento.

\section{DISCUSSÃO}

M uito se tem discutido sobre o papel da fisioterapia no tratamento pós-cirúrgico do câncer de mama.

Camargo e $\mathrm{M} \mathrm{arx}^{24}$, Serravalle ${ }^{19}$, Kaplan ${ }^{25}$ e $\mathrm{N}$ ewsome ${ }^{26}$ citam a importância da cinesioterapia no pós-operatório imediato e posteriormente. No entanto, não se encontra na literatura referências a trabalhos com exercícios passivos combinados com ativo-livres, como também são raramente encontrados trabal hos de campo.

No protocolo apresentado e efetivamente aplicado no IGM (Instituto de Ginecologia e M astologia), foi realizado um trabalho com exercícios passivos, ativolivres ea pompage, promovendo desta forma um contato maior do fisioterapeuta com a paciente, proporcionando um tratamento mais completo e eficaz.

$Q$ uando se efetua a comparação entre os G rupos A e $B$, observa-se uma diferença significativa entre os graus de limitação da AD M e da evolução de linfedema.

O s resultados do presente estudo confirmam os dados já obtidos por outros autores, como Sasaki e Lamari'27, Camargo e M arx ${ }^{24}$, Bruno et $a^{28}$, Kaplan ${ }^{25}$ e $\mathrm{N}$ ewsome ${ }^{26}$, de que a reabilitação da função do membro superior e a diminuição das complicações no pósoperatório em mulheres mastectomizadas é obtida a partir da introdução da fisioterapia.

Sasaki e Lamari27, em seu trabalho utilizaram apenas cinesioterapia (exercícios ativos e ativos associados contra a gravidade) e obtiveram os resultados em 40 sessões (ADM para realizar as AVD);

$\mathrm{N}$ o protocolo apresentado neste estudo as pacientes precisaram, em média, de 10 sessões. As variações desse número foram dependentes da disponibilidade da paciente para realizar em casa os exercícios orientados.

No presente estudo ocorreu menor evolução para linfedema nas pacientes que seguiram as orientações do protocolo aceito no serviço, fato não avaliado no estudo de Sasaki e Lamari27.

0 protocolo realizado no IG M requer colaboração e envolvimento total das pacientes, uma vez que todas elas foram orientadas a realizar, em casa, exercícios a partir do manual fisioterapêutico para pós-operatório em pacientes submetidas à mastectomia, não descrito nos estudos por Sasaki e Lamarii ${ }^{27}$, B runo et al ${ }^{28}$, Kaplan ${ }^{25}$ e $\mathrm{N} \mathrm{ewsome} \mathrm{e}^{26}$.

Bruno et $a^{28}$, apresentam um programa de exercícios a serem realizados no pós-operatório, porém não citam se esse programa foi efetivamente aplicado a algum grupo de pacientes.

Conforme descrito nas figuras deste trabalho, bem como em suas respectivas análises descritivas, fica evidente que o protocolo aqui apresentado e aplicado, traz resultados significativos, no ganho da ADM. Possivelmente traz também benefício na prevenção ou diminuição de um eventual linfedema.

As pacientes do Grupo $B$, que apresentaram limitação de ADM e linfedema, foram convidadas a retornarem para a fisioterapia, e serão acompanhadas pelo departamento de fisioterapia do IGM até uma possível alta. 
0 presente estudo, se ampliado, poderá mostrar outros benefícios do tratamento fisioterapêutico nas pacientes com câncer de mama operadas através da técnica de $M$ adden, devendo ainda ser avaliados os benefícios em outras técnicas cirúrgicas.

\section{CONCLUSÃO}

A intervenção precoce da fisioterapia, aplicada ainda no ambiente hospitalar, não só ajuda a prevenir as complicações pós-cirúrgicas, como também reabilita as pacientes mais cedo para as atividades da vida diária (AVD ), e ainda, permite a elas que possam se valer da colaboração e do incentivo da equipe médica para 0 tratamento.

- 0 protocolo apresentado nesteestudo mostrou-se eficaz para as pacientes pós-mastectomizadas, uma vez que: As pacientes retornaram a realizar atividades da vida diária com uma média de 10 sessões.

- Apesar de não ter sido analisado estatisticamente (pequeno número de pacientes no grupo $B$ ), houve uma menor incidência de linfedema no grupo que realizou 0 tratamento, quando comparado com o grupo controle (Grupo $A=10 \%$ e Grupo $B=36 \%$ ).

- As pacientes que se submeteram ao tratamento e que, na primeira avaliação, não atingiam $180^{\circ}$ de flexão e abdução da articulação gleno-umeral do membro superior contra-lateral à cirúrgica, obtiveram um ganho total, na maioria dos casos, do grau de flexão e abdução.

Todas as integrantes deste estudo continuarão sendo acompanhadas pelo departamento de fisioterapia do IG M , pelo prazo de seis meses, para observação posterior do quadro clínico.

\section{REFERÊNCIAS}

1. Beahrs OH , H enson DE, H utter RVP, Kennedy BJ. M anual for staging of cancer. 4th ed. Philadel phia: American Joint Committee on Cancer; 1992.

2. Instituto Nacional de Câncer; M inistério da Saúde, Coordenação de Programas de C ontrole de C âncer. 0 problema do câncer no Brasil. 4a ed. Rio deJ aneiro (Brasil): Pro-O nco; 1997.

3. Instituto $\mathrm{N}$ acional de $\mathrm{C}$ âncer; $\mathrm{M}$ inistério da Saúde. Câncer demama. Rev BrasC ancerol. 2001;47(1):9-19.

4. Ministério daSaúde;U nião Internacional Contrao Câncer.TN M Classificação dostumoresmalignos. Braślia(Brasil); 1989.

5. D rinkwater N R, Sugden B. In: Fundação O nconcentro de São Paulo; U nião Internacional C ontra o Câncer. M anual de onconlogia clínica. 2a ed. bras. São Paulo;1997.
6. Abreu $E, K$ oifman $S$. Fatores prognósticos no câncer da mama feminina. Rev BrasC ancerol. 2002;48(1):113-31.

7. LeiteRC, $O$ liveiraC, Ribeiro L. $C$ âncer demama prevenção etratamento. São Paulo: Ediouro; 2002.

8. Singhal H. Breast cancer evaluation. M ed J. 2001;2(9):1-10.

9. M ontoro AF. Prevenção e detecção do câncer demama. São Paulo: UniversidadedeSão Paulo; 1979.

10. Falzoni R. M anual depatologiamamária: roteirosanatomocirúrgicos das lesões malignas e pré-malignas. São Paulo: Audichromo; 1996.

11. Eckhardt S, Borden EC. In: Fundação O nconcentro de São Paulo; U nião I nternacional C ontra o Câncer. M anual deonconlogia clínica. 2a ed. bras. São Paulo;1997.

12. Sherman CD J r, H ossdeld D K . In: Fundação O nconcentro deSão Paulo; União Internacional Contrao C âncer. M anual deonconlogia clínica. 2a ed. bras. São Paulo;1997.

13. Jatoi I. Clin Cir Am N orte. 1999;79(5).

14. Pinelli D, Vaiana R, Bianchi A, Alghisi A, Ragni F, Braga $M$. Attuali indicazioni alla mastectomia radicale secondo $M$ adden nella nostra esperienza. $M$ inerva $C$ hir. 1997;52(4):403-10.

15. Savlov E. Câncer demama. In: Rubin P. M anual declínica oncológica. 4aed. São Paulo: Sarvier; 1977.

16. Silva S, Zurrida OE. Câncer de mama: um guia para médicos. São Paulo: Atlântica; 2000.

17. Veronesi U. Cirugía oncológica. Buenos Aires: M édica Panamericana; 1991.

18. D umortier A, Le Vu B, G uillaume M V, M ouriesse $H$, Barreau-Pouhaer L. Efficacité du massageet la mobilisation du membresupérieur aprèstraitement chirurgical du cancer du sein. Bull Cancer.1997;84(10):957-61.

19. SerravalleN . Fisioterapia em mastectomizadas. FisioTerap. 2000;4(18):20-1.

20. G uirro E, G uirro G. Fisioterapia dermatomo-funcional. 3a ed. São Paulo: M anole; 2002.

21. M arquesAP. M anual degoniometria. São Paulo: M anole; 1997.

22. Bienfait M . Estudo etratamento do esqueleto fibroso: fáscias epompagens. 2a ed. São Paulo; 2001.

23. D oria Filho U . Introdução à bioestatística: para simples mortais. 3a ed. rev. ampl. São Paulo: N egócio Editora; 1999.

24. C amargo M C, M arx AG . Reabilitação física no câncer de mama. São Paulo: Roca; 2000.

25. Kaplan RJ. Cancer rehabilitation. M ed J . 2001;2(8):2-11.

26. N ewsomeRE. Breast cancer. M ed J. 2002;3(6):2-10.

27. Sassaki T, Lamari N M . Reabilitação funcional precocepós mastectomia. H B Cient. 1997;4(2):121-7.

28. Bruno AA, M asiero D, Secco M FM, Wasserstein S, Chamlian TR. Padronização deconduta na reabilitação póscâncer demama. J Bras M ed. 2000;78(5):86-92. 